\title{
Protocol
}

\section{An evaluation of the effectiveness of a community-based parenting empowerment program to improve nurturing care of young children in Kenya and Zambia}

\author{
Patricia K. Wekulo ${ }^{1}$, Elizabeth K. Murage ${ }^{1}$, Hermann P. Donfouet ${ }^{1}$, Silas O. Onyango ${ }^{1}$, \\ Kenneth O. Okelo ${ }^{1 *}$, Milka N. Wanjohi ${ }^{1}$, Dawn Murdock ${ }^{2}$, George Nyamor ${ }^{3}$, Kelvin Munsongo ${ }^{4}$ \\ ${ }^{1}$ Maternal and Child Wellbeing Unit, African Population and Health Research Center, Nairobi, Kenya \\ ${ }^{2}$ Episcopal Relief and Development, New York, USA \\ ${ }^{3}$ Anglican Development Service -Nyanza, Kisumu, Kenya \\ ${ }^{4}$ Zambian Anglican Council Outreach Program, Lusaka, Zambia
}

Received: 02 September 2018

Revised: 06 June 2019

Accepted: 14 June 2019

\section{*Correspondence:}

Dr. Kenneth O. Okelo,

E-mail: kokelo@aphrc.org

Copyright: (c) the author(s), publisher and licensee Medip Academy. This is an open-access article distributed under the terms of the Creative Commons Attribution Non-Commercial License, which permits unrestricted non-commercial use, distribution, and reproduction in any medium, provided the original work is properly cited.

\section{ABSTRACT}

Background: Investing in parents and children during the critical period between birth and five years of a child's life can have long-lasting benefits in the life of the child. Recently, the 2016 Lancet Series estimated that 250 million children aged less than five years in low- and middle-income countries (LMICs) are at risk of not reaching their developmental potential. Over $66 \%$ of these, who live in sub-Saharan Africa and are at risk due to poor nutrition, exposure to poverty, high HIV prevalence as well as under stimulation in the home environment.

Methods: The study will employ a cluster-randomized control trial design and will use a mixed-methods approach combining quantitative and qualitative methodologies (Concurrent Triangulation Design). This will be a two-arm study, where the first arm will participate in the parenting empowerment program implemented by the faith-based organizations, while the second (control) will not receive the parenting interventions. A total of 510 mother/primary caregiver-child dyads will be recruited into this study. We will estimate the causal effect of the intervention using mixed linear models and the Difference-in-Differences (DID) estimator.

Conclusions: This implementation research will provide greater scientific rigor and a deeper process and outcome evaluation including measurement of child development outcomes. The findings will be useful for early childhood practitioners, multilateral stakeholders and funders as they provide information on the factors to consider in the implementation of high quality ECD interventions.

Trial Registration: Ethical approval: ESRC P467/2018 and IRB No.00005948).

Keywords: Child development, Early childhood, Nurturing care, Stimulation, Parenting empowerment

\section{INTRODUCTION}

Investing in parents and children during the critical period between birth and five years of a child's life can have long-lasting benefits in the life of the child. Recently, the 2016 Lancet Series on advancing early childhood development estimated that 250 million children aged less than five years in low- and middle- income countries (LMICs) are at risk of not reaching their developmental potential. ${ }^{1}$ Over $66 \%$ of these, who live in sub-Saharan Africa (SSA), are at risk due to poor nutrition, exposure to poverty, high HIV prevalence as well as under stimulation in the home environment. These factors adversely affect their cognitive, physical, and socio-emotional development and consequently deter from achieving their developmental potential. ${ }^{2}$ Children 
living in rural and poor urban areas are the most affected as they lack basic rights of survival, development and protection, as well as marginalization and exclusion from essential services such as health care, clean water, proper sanitation, and quality education. ${ }^{3,4}$ The Lancet Series recommended integrated interventions which combine nutrition, responsive child feeding and child stimulation, as well as the expansion of high quality and cost-effective early childhood development (ECD) programs as they result in significant gains..$^{5}$ Further, the Lancet Series recommends parenting programs as an effective means to support the capacity of primary caregivers to provide the proper early learning environments that children need.

Parenting programs can strengthen parents' ability to manage a child's behaviour, support socio-emotional development, and reduce child neglect and abuse. Studies of parental interventions have reported improved developmental outcomes of children, as well as mothers' knowledge on child development. ${ }^{1}$ For instance, a 12session integrated parenting interventions delivered by non-professional community members was shown to improve child development and maternal wellbeing in rural Uganda. ${ }^{2}$ A systematic review of parenting programs for young children in low- and middle-income countries revealed that parenting sessions improved parents' ability to psychologically stimulate their children, improved responsivity in parenting as well provision of improved care for young children. ${ }^{3}$ Parental sessions are paramount and thus an avenue through which issues that children face can be addressed. Despite the profound role played by parental empowerment programs in improving caregivers' ability to promote and support their children's development, little has been done to promote such programs in sub-Saharan African countries like Kenya and Zambia.

In response to this, beginning in 2012, Episcopal Relief and Development and its implementing partner in Zambia, Zambian Anglican Council Outreach Programmes (ZACOP), launched an integrated ECD program aimed at strengthening families so young children can thrive. Rural communities, where many families are affected by HIV/AIDS and other vulnerabilities, are engaged to work with vulnerable families with children under the age of three years, through local ECD committees who oversee ECD volunteers and connect to other stakeholders. Trained ECD promoters who are a new type of local volunteer (rather than community health workers) lead the main parenting empowerment activities. The parenting empowerment component focuses on responsive care and stimulation by the primary caregiver, and the primary caregivers' own well-being through support and learning groups, as well as ECD home visits. 'Primary caregiver' is used to refer to the person serving in the main parenting role in the home; some fathers and other male relatives also serve as primary caregivers. Where fathers are present in a household, the program specifically engages them or other male secondary primary caregivers in some activities.

The integrated program addresses nurture and stimulation of under-three-year-old children, child, and maternal/ family health, nutrition, and livelihoods issues through a combination of direct project activities and linking with available services. Based on those findings, Episcopal Relief and Development and its partners is adding the parenting empowerment approach to their work in western Kenya. In 2018, the Anglican Development Services (ADS)-Nyanza is adapting the model and starting at small scale. ADS will work with 429 families in Onjiko/Awasi Ward, Nyando Sub-County of Kisumu County. The program will engage local faith leaders and groups as catalysts for change in parenting and early childhood development.

\section{Overall purpose}

The overall purpose of the parenting empowerment program is two-fold:

- Build evidence for a high impact, cost-effective parenting empowerment model for scaling up by Episcopal Relief \& Development and its implementing Anglican partners, other faith-based organizations, $\mathrm{CBO} / \mathrm{NGOs,} \mathrm{and} \mathrm{government} \mathrm{entities}$ in sub-Saharan Africa (SSA).

- Contribute to the evidence base and learning in the ECD sector's nurturing care framework for children 0-3 years, focusing on parenting responsive care and stimulation among vulnerable families, including those affected by HIV and AIDS.

The African Population and Health Research Center (APHRC) will conduct an external evaluation of the parenting empowerment program. The goal of the external evaluation by the APHRC is to assess the efficiency of the parenting empowerment program, and establish the impact of the program on specific caregiver and child outcomes. This evaluation will provide information on the effectiveness of the program in achieving its objectives. The main objectives of the evaluation are outlined in the next section.

\section{Implementation research objectives}

The study will involve an impact evaluation, as well as monitoring and evaluation (M\&E) activities (to be referred to as "process evaluation" henceforth). This section covers the objectives to be achieved during the process and outcome evaluation within the study.

\section{Process evaluation}

The process evaluation seeks to establish factors affecting implementation, the process of implementation and the results of implementation. We intend to establish what, why and how the proposed intervention works in "real 
world" settings, and to test approaches to improve them. ${ }^{1}$ The objectives of this component of the evaluation are to:

- Assess ECD volunteer training, mentoring support and supervision to determine what suffices for quality work;

- Assess processes for selection of the most vulnerable families which ensures the target population is reached and equity of ECD access provided to low-income, highly vulnerable children;

- $\quad$ Assess program strategies and practices to validate what works to achieve parenting behaviour change and improved child development; also including specifically:

- fathers' and other male primary caregivers' behaviour change, and women's and men's attitudes about gender roles in parenting;

- role of faith leaders in parenting social and behaviour change (focused on fathers' role, positive discipline/reduction of harsh punishment, and child rights/protection);

- Determine which major project activities could be effectively monitored as markers for quality assurance and control;

- Determine which best practices for adapting and scaling the model to other rural African country contexts could be documented;

- Establish facilitators and barriers to program implementation;

- $\quad$ Assess cost and cost-effectiveness of the program model for parenting empowerment.

\section{Outcomes}

The process evaluation outcome variables will include acceptability, adoption, appropriateness, feasibility, fidelity, implementation cost, coverage, and sustainability. The primary outcomes for the outcome evaluation will include level of engagement of faith leaders, ECD volunteer effectiveness, parenting attitudes and behaviour change on responsive care and stimulation, primary caregiver well-being, primary caregiver savings and loans group participation, parenting behaviour change on essential health and nutrition actions. The secondary outcome will be child developmental outcomes

\section{Outcome evaluation}

The overall objective of the outcome evaluation is to establish if the model strengthens the capacities of families and increases adoption of high-impact behaviours to improve primary caregiver-child interaction, and nurturing care and stimulation (early learning) so that young children reach their full developmental potential. Outcomes will be measured after 12 months of participation in project activities (midterm), and after 24 months of participation (endline).

The specific objectives are as follows:
- To establish the impact of the program on parents/primary and secondary (particularly fathers) caregivers' responsive care and stimulation practices with their children aged $0-3$ years;

- To determine the effect of the program on primary caregivers' well-being (mental health and stress levels), sense of parenting efficacy and connections to services;

- To determine the effect of the program on primary caregivers' practice of essential health and nutrition actions;

- To establish the impact of the program on families' economic well-being through savings and loan groups and business education;

- To establish whether the program strengthens the capacities of ECD promoters, faith and other leaders to manage and sustain $\mathrm{ECD} /$ positive parenting activities and norms in their communities;

- To determine the effect of the program on children's developmental outcomes.

\section{METHODS}

In Kenya, the evaluation will employ a cluster quasiexperimental design. We will use both quantitative and qualitative data collection methods to obtain information on the different aspects of the study. The villages or clusters will be purposively selected, taking into consideration such factors as poverty levels and number of families in order to make them as comparable to each other as possible. We will select three clusters from each of the two sub-locations within the project implementation sites, and six clusters from a third sublocation where there are no program activities.

In Zambia, the evaluation will employ a clusterrandomized control trial design (see flow chart in Figure 1) and use a mixed-methods approach. We will use both quantitative and qualitative data collection methods in a concurrent triangulation design to obtain information on the different aspects of the study. The concurrent triangulation design will allow us to corroborate and support the results obtained through different methods (qualitative and quantitative), and will enhance the external validity of the findings. ${ }^{4}$ Furthermore, by using a mixed methods approach, we will endeavour to reduce the weaknesses of each type of data collection method, and enrich our understanding of the issues being studied. ${ }^{5}$

Ten communities within the project site are considered the clusters. Within the study district, communities will be randomly selected from project sites, and stratified at the community level to reduce potential contamination between study arms. There will be five communities from one ward allocated to the intervention arm and five communities located in a different ward allocated to the control arm. We will use a buffer zone, as well as restricted allocation to ensure that contamination is minimized to the extent possible. 


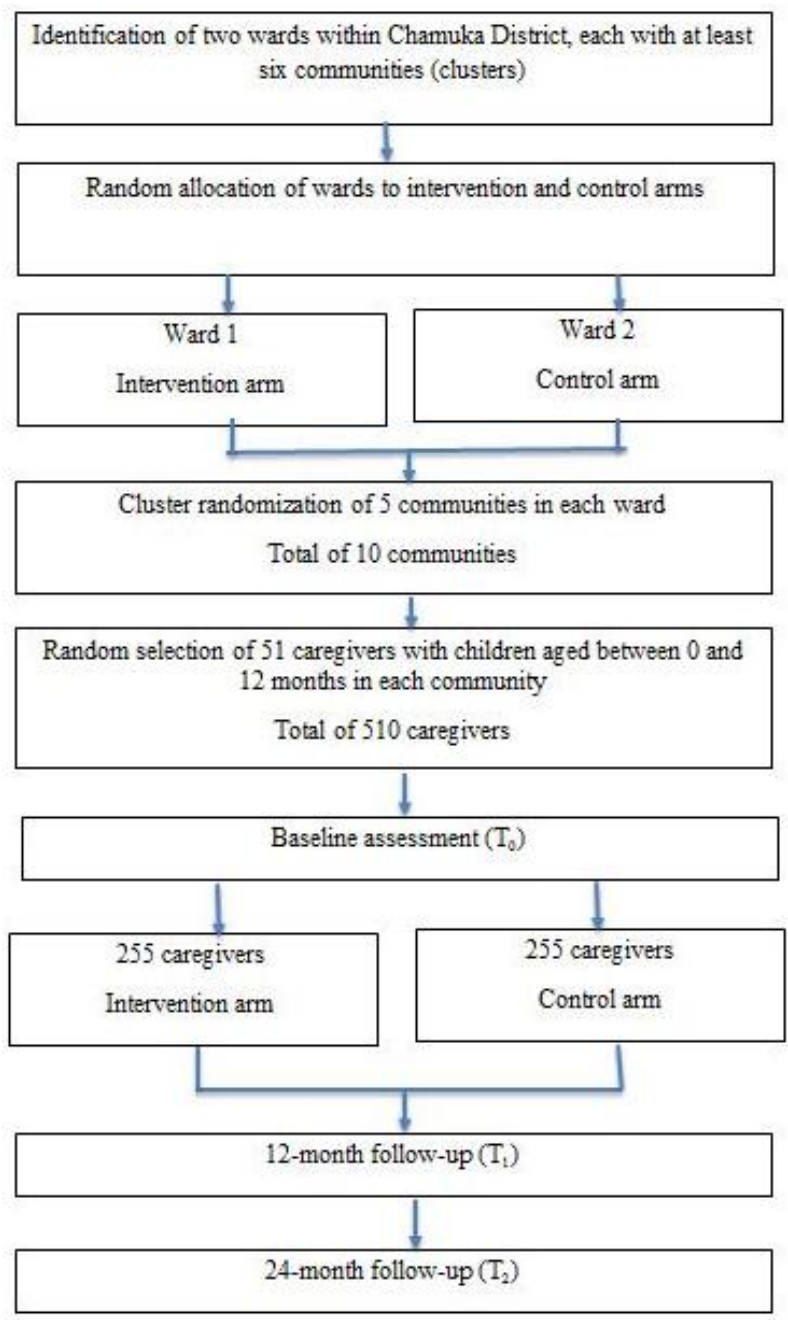

Figure 1: Flow chart of the process followed in the cluster-randomized control trial.

\section{Description of the intervention}

Through a participatory process involving local stakeholders and leaders, the most vulnerable families in a community are identified and invited to participate. The program has an intensive 24-month parent participation timeframe, within an implementation period of approximately 30-36 months. This allows 3-6 months for stakeholder and community mobilization, baseline selection and training of ECD volunteers, and family selection before the groups start meeting. After the cycle is completed, sometime is set aside for an evaluation and organizing to start the cycle with new families.

Primary caregivers participate in ECD volunteerfacilitated monthly parenting support and learning group sessions, and ECD home visits by the volunteers for 24 months (a total of approximately 24 group meetings and 24 ECD home visits). Group sessions are expected to enhance social motivation to participate and provide peer support and learning. At month 13, the primary caregiver groups start participating in training to form and manage their own savings \& loan groups, with Episcopal Relief \&
Development's Savings with Education (SwE) methodology. These groups then become the vehicle for continued member-managed activities, and the ECD volunteers move on to new families forming new primary caregiver support and learning groups. The health and nutrition components are mainly provided by strengthening connections to community health workers and their integrated community case management work, as well as health staff and the health system. ECD volunteers promote families' participation in growth monitoring, health and nutrition education, cooking classes with local ingredients and immunization campaigns. Some additional activities will vary according to the project context. For example, families will have the opportunity to learn and establish kitchen gardens to improve their nutrition and generate income from selling the surplus.

Trained ECD promoters who will facilitate learningaction dialogues and peer support with primary caregivers through picture-based social and behaviour change communication (SBCC) will deliver the integrated early child stimulation intervention. Each ECD promoter will be responsible for between 7 and 13 households (primary caregivers, children, and primary caregiving environment) in each community. Home visitations will be conducted on a monthly basis during which promoters will discuss various issues with the primary caregivers related to parenting skills, health, nutrition, primary caregiving environment and water, sanitation and hygiene. These home visitations will be carried out in a participatory manner-using social and behaviour change communication, and will aim to improve primary caregivers' knowledge, awareness, and behaviour in line with the behaviour-change strategy. The ECD promoters are central to this process not only because they are conversant with the local context, but also as a way of utilizing local resources and building capacity to promote sustainability of the project.

Based on those findings, Episcopal Relief and Development and its partners is adding the parenting empowerment approach to their work in western Kenya. In 2018, the Anglican Development Services (ADS)Nyanza is adapting the model and starting at small scale. ADS will work with 429 families in Onjiko/Awasi Ward, Nyando Sub-County of Kisumu County. The program will engage local faith leaders and groups as catalysts for change in parenting and early childhood development.

Over the years, an aspect of involvement of religious organizations in ECD has been missing in implementation of ECD activities. The ADS model emphasizes faith leaders' (clergy and lay readers) engagement in promotion of ECD, focusing on the wider community and addressing the challenges around uptake and perception. The project will therefore utilize the rich church structure and its wide reach for ECD program delivery to community leaders, during weekly Sunday services, during faith leaders' meetings, during pastoral 
visits to households and during cell group meetings. The organization will facilitate formation of a faith leaders' consortium aimed at promoting and sustaining social and behaviour change for ECD and positive parenting, in communities of implementation. The consortium will hold monthly meetings to share experiences and progress.

\section{Inclusion and exclusion criteria}

\section{Inclusion criteria}

All primary caregivers of children aged between zero and twelve months participating in the intervention program will be eligible for inclusion in the evaluation study. Families in the intervention program are selected on the basis of their vulnerability status (for example, health and nutrition indicators, HIV exposure, poverty levels). They will be long-term residents (more than one year) of the study area.

\section{Exclusion criteria}

Any short-term residents (less than one year) will be excluded from the study. Caregivers of children above the age of three years will also be excluded from the study. Also excluded will be women (or men) incapable of providing consent (e.g. those with mental impairments)

\section{Ethical considerations}

The study will be conducted after internal review by APHRC's internal scientific review committee has been completed and ethics approval from AMREF's Ethical and Scientific Review Committee has been received.

The research team has undergone NIH training on protecting human research participants (copies of certificates attached with this application). We shall seek informed consent from all study participants. For those who cannot read, the information sheet will be read to them in their local language and they will be asked to provide a thumb print to signify their consent. All survey data will be collected in privacy and will be treated with confidentiality.

\section{Data collection tools}

\section{Primary caregiver questionnaires}

Primary caregiver knowledge, attitudes, and practices (KAP) with regards to responsive care and early child stimulation were measured using a set of questions that cover topics such as appropriate feeding practices and play and learning activities likely to promote holistic development in children. Other tools used to collect information on parents' demographic characteristics and caregiver wellbeing were the socio-demographic questionnaire, the Global Indicator Outcome Survey (GIOS) and the Parental Stress Index (PSI).

\section{Fathers' questionnaire}

Fathers' (who are co-parents, i.e., not serving as the primary caregiver) parenting practices were assessed by their self-report of their responsiveness and ageappropriate play and learning activities with the child at home.

\section{Ages and stages questionnaire - third edition (ASQ-3)}

Children's developmental outcomes in terms of their gross and fine motor skills, language, socio-emotional and physical development were measured using the ages and stages questionnaire - third edition (ASQ-3).

\section{Qualitative interviews}

Interviews (focus group discussions [FGDs], in-depth interviews [IDIs] and key informant interviews [KIIs]) with primary caregivers, fathers, community health workers/volunteers (CHW/Vs), ECD promoters, project staff, community leaders, faith leaders and health staff to establish their perceptions about the MTM program.

\section{RISE observation checklist}

In order to assess the family home environment and primary caregiver-child interaction, the study will use the draft observation tool in development at the time (May 2018) by the Stellenbosch University team contracted by the Conrad N. Hilton Foundation (CNHF). The observation tool had twelve (12) items that the field interviewers used to assess the interaction between the child and the caregiver, as well as whether or not the home environment supports play and stimulation activities.

\section{Data processing and analysis}

\section{Process evaluation}

Qualitative interviews will be audio recorded and transcribed verbatim in English. The transcripts will then be coded in NVivo version 10, after which thematic analysis will be done in line with the key questions. Analysis across all transcripts will be done thematically.

Costing data will be summarized and described for each cost item and sensitivity analysis will be carried out to estimate the cost the ECD intervention. We will determine the cost of implementing the ECD integrated program.

\section{Outcome evaluation}

A first set of analyses will consist of descriptive statistics. For instance, descriptive analysis will be done to establish the number of primary caregivers participating in the savings and loans groups as well as their frequency of participation in the groups. We will also establish the 
proportion of ECD promoters, community leaders and faith leaders whose practices conform to the set guidelines.

\section{Econometric models}

We will use the difference-in-differences (DID) methods to estimate the causal impact of the ECD model since the outcomes of the control and intervention groups are measured at two different time periods (at baseline - once before the implementation of the ECD program, and midline/end line - second time after the implementation of the ECD program). In the analysis, we will take into consideration differences in parenting behaviour change according to different characteristics such as presence/absence of both parents, and gender and age of the primary caregiver. This will allow us to detect similarities and/or differences between subjects' characteristics across the different groups. In other words, we will compare some baseline measurements between the control group and intervention group using the t-test adjusted for clustering at the community level for continuous variables, and cluster-adjusted chi-square for binary variables. Hence, with the DID we will estimate the treatment effect where we consider the baseline and midline, and also when we cogitate for the baseline and end line.

A sensitivity analysis will also be done by using a DID multilevel model. Specifically, we will consider a twolevel DID mixed-effects model. In this two-level model, a set of random effects at cluster level (the second level) is included to control for cluster-specific random variation, and a random-error term at the observation level (the first level) to control for within-cluster variation.

Given the longitudinal nature of the survey, we will also investigate the possible linear change in the outcome of the ECD program according to the length of the implementation of the ECD program. Comparing levels of parenting behaviour change and child development after 12 months of participation (midterm) versus after 24 months (endline) could be helpful in examining whether the ECD program sets children on a trajectory of positive development.

Individual items on the ASQ-3 will be reviewed for missing data, as well as for floor and ceiling effects. In the case where more than $10 \%$ of the responses on the items are missing, the data will be excluded from further analysis. The time taken to complete the assessment will be calculated. We will also calculate the totals by summing the scores of the items included in the tool to establish children's performance levels at baseline and at endline. This will enable us to identify child development domains that have acceptable variability.

Qualitative interviews will be audio recorded and transcribed verbatim in English. The transcripts will then be coded in NVivo version 10, after which, thematic analysis will be done in line with the key questions. Analysis across all transcripts will be done thematically.

The audio-taped interviews will be retained for a twoyear period after the end of the study, after which they will be disposed of through deletion. In addition, any records stored on a computer hard drive will be erased using commercial software applications designed to remove all data from the storage device.

\section{DISCUSSION}

The implementation research by the APHRC will provide greater scientific rigor and a deeper process and outcome evaluation including measurement of child development outcomes. Over the course of the project implementation, monitoring, evaluation and learning (MEL) work within the process evaluation led by the APHRC will contribute to Episcopal Relief and Development and its partners making quality improvements on the program and will support and inform scale-up with quality. Episcopal Relief and Development will share this model and package of training and curricula with some of its other African partners for replication and adaptation as part of their rural integrated programming.

The implementation research will assess the results of the program's strategies and activities, the processes used in carrying out the program, the contextual factors that affect those processes, the cost-effectiveness of the program model, and how to promote sustainability, adaptation and scaling up with quality of the program model in rural SSA. The research findings will provide government stakeholders with knowledge for planning future programs, strategic decisions and policy-making. The findings are useful for early childhood practitioners, multilateral stakeholders and funders as they provide information on the factors to consider in the implementation of high quality ECD interventions.

Funding: Episcopal Relief \& Development with a grant from the Conrad N. Hilton Foundation and funding from other donors

Conflict of interest: None declared

Ethical approval: The study was approved by the Amref Health Africa Ethics and Scientific Review Committee in Kenya (ESRC P467/2018) and ERES Converge in Zambia (IRB No.00005948)

\section{REFERENCES}

1. Black MM, Walker SP, Fernald LCH, Andersen CT, DiGirolamo AM, Lu C, et al. Early childhood development coming of age: science through the life course. Lancet. 2017;389:77-90.

2. Britto PR, Lye SJ, Proulx K, Yousafzai AK, Matthews SG, Vaivada $\mathrm{T}$, et al. Nurturing care: promoting early childhood development. Lancet. 2017;389:91-102. 
3. Kimani-Murage EW, Kimiywe J, Kabue M, Wekesah F, Matiri E, Muhia N, et al. Feasibility and effectiveness of the baby friendly community initiative in rural Kenya: Study protocol for a randomized controlled trial. Trials. 2015;16(1).

4. Ambey R, Gaur A, Gupta R, Patel G. Urban poor children. Australas Med J. 2013;6(6):341-3.

5. Richter L, Daelmans B, Lombardi J, Heymann J. Investing in the foundation of sustainable development: pathways to scale up for early childhood development. Lancet. 2017;389(10064):103-18.

6. Engle PL, Fernald LCH, Alderman H, Behrman J, O'Gara C, Yousafzai A, et al. Strategies for reducing inequalities and improving developmental outcomes for young children in low-income and middle-income countries. Lancet. 2011;378(9799):1339-53.

7. Walker SP, Powell CA, Grantham-McGregor SM, Himes JH, Chang SM. Nutritional supplementation, psychosocial stimulation, and growth of stunted children: The Jamaican study. Am J Clin Nutr. 1991;54(4):642-8.

8. Yousafzai AK, Aboud F. Review of implementation processes for integrated nutrition and psychosocial stimulation interventions. Ann N Y Acad Sci. 2014;1308(1):33-45.

9. Engle PL. Parenting Programme for Child Development. Source J Heal Popul Nutr. 2007;2525(11):1-2.

10. Engle PL, Black MM, Behrman JR, Cabral de Mello M, Gertler PJ, Kapiriri L, et al. Strategies to avoid the loss of developmental potential in more than 200 million children in the developing world. Lancet. 2007;369:229-42.
11. Engle P, Menon P, Haddad L. Care and nutrition: concepts and measurement. World Dev. 1999;27(8):1309-37.

12. Digirolamo AM, Stansbery $\mathrm{P}$, Lung'aho $\mathrm{M}$. Advantages and challenges of integration: Opportunities for integrating early childhood development and nutrition programming. Ann N Y Acad Sci. 2014;1308(1):46-53.

13. Gowani S, Yousafzai A, Armstrong R. Cost effectiveness of responsive stimulation and nutrition interventions on early child development outcomes in Pakistan. Ann N Y Acad Sci. 2014;1308:149-61.

14. Ministry of Health. Kisumu County Health at a Glance. 2015;(2014):2015.

15. Kenya National Bureau of Statistics (KNBS); ICF Macro. Kenya Demographic and Health Survey 2014. Heal San Fr. 2014;1-314.

16. County K. First county integrated development plan 2013-2017; 2013.

17. Hsiao C, Richter L, Makusha T, Matafwali B, van Heerden A, Mabaso M. Use of the ages and stages questionnaire adapted for South Africa and Zambia. Child Care Health Dev. 2017;43(1):59-66.

18. Squires J, Bricker D. Ages \& Stages Questionnaires. Third Edition. (ASQ-3): A Parent-Completed Child-Monitoring System. Paul Brookes Publication Co; 2009.

Cite this article as: Wekulo PK, Murage EK, Donfouet HP, Onyango SO, Okelo KO, Wanjohi MN, et al. An evaluation of the effectiveness of a community-based parenting empowerment program to improve nurturing care of young children in Kenya and Zambia. Int J Clin Trials 2019;6(3): 122-8. 